This item was submitted to Loughborough's Research Repository by the author.

Items in Figshare are protected by copyright, with all rights reserved, unless otherwise indicated.

\title{
Comparison of biomechanical characteristics between male and female elite fast bowlers
}

PLEASE CITE THE PUBLISHED VERSION

https://doi.org/10.1080/02640414.2018.1522700

\section{PUBLISHER}

(C) Taylor \& Francis

\section{VERSION}

AM (Accepted Manuscript)

\section{PUBLISHER STATEMENT}

This is an Accepted Manuscript of an article published by Taylor \& Francis in Journal of Sports Sciences on 22 September 2018, available online: http://www.tandfonline.com/10.1080/02640414.2018.1522700.

\section{LICENCE}

CC BY-NC-ND 4.0

\section{REPOSITORY RECORD}

Felton, Paul, S.L. Lister, Peter J. Worthington, and Mark A. King. 2019. "Comparison of Biomechanical Characteristics Between Male and Female Elite Fast Bowlers”. figshare. https://hdl.handle.net/2134/35197. 


\title{
Comparison of biomechanical characteristics between male and female elite fast bowlers

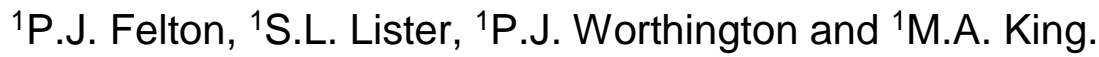

${ }^{1}$ School of Sport, Exercise and Health Sciences, Loughborough University, Leicestershire, LE11 3TU, UK

\begin{abstract}
This study investigated ball release speed and performance kinematics between elite male and female cricket fast bowlers. Fifty-five kinematic parameters were collected for 20 male and 20 female elite fast bowlers. Group means were analysed statistically using an independent samples approach to identify differences. Significant differences were found between: ball release speed; run-up speed; the kinematics at back foot contact (BFC), front foot contact (FFC), and ball release (BR); and the timings between these key instants. These results indicate that the female bowlers generated less whole body linear momentum during the run-up than the males. The male bowlers also utilised a technique between BFC and FFC which more efficiently maintained linear momentum compared to the females. As a consequence of this difference in linear momentum at FFC, the females typically adopted a technique more akin to throwing where ball release speed was contributed to by both the whole body angular momentum and the large rotator muscles used to rotate the pelvis and torso segments about the longitudinal axis. This knowledge is likely to be useful in the coaching of female fast bowlers although future studies are required to understand the effects of anthropometric and strength constraints on fast bowling performance.
\end{abstract}

Keywords: cricket, ball release speed, gender differences, kinematics

\section{INTRODUCTION}

Cricket is an international sport which is played professionally by both males and females. In a cricket team, the majority of bowlers are normally classified as fast bowlers. Fast bowlers attempt to deliver the ball towards the batter as fast as possible to reduce the time the batter has to interpret the delivery and play the appropriate stroke. Coaching therefore, focuses on increasing ball release speed by improving bowling technique.

Several studies have investigated the relationships between ball release speed and various technique characteristics (Davis and Blanksby, 1976a, 1976b; Elliott et al., 1986; Foster et al., 1989; Burden and Bartlett, 1990a, 1990b; Portus et al., 2004; Worthington et al., 2013a). All these studies, however, have used male fast bowlers as participants. Initial studies used linear correlations to determine whether a particular aspect of bowling technique was independently linked to ball release speed (Davis and Blanksby, 1976a, 1976b; Elliott et al., 1986; Foster et al., 1989; Burden and Bartlett, 1990a, 1990b; Portus et al., 2004). More recently, Worthington et al. (2013a) used a regression technique and found that four technique parameters were capable of explaining $74 \%$ of the observed variance in ball release speed for 20 elite fast bowlers. The results indicated that the fastest bowlers have a quicker run-up and maintain a straighter front knee throughout the front foot contact phase. The fastest bowlers were also observed to exhibit larger amounts of upper trunk flexion between front foot contact and ball release, and to delay the onset of arm circumduction. This suggests that the optimal technique to generate higher ball release speeds for male fast bowlers is one in which whole body linear momentum is generated via the run-up and then converted to whole body angular momentum about the mass centre, using the front leg to brake the lower half of the body and accelerate the top half towards the target (Worthington et al., 2013a). The larger amounts of upper trunk flexion may represent a more effective conversion of linear momentum to angular momentum. The delay in 
the bowling arm is a consequence of this increased amount of upper trunk flexion, as more trunk flexion requires a delayed bowling arm in order to release the ball towards the same target (Worthington et al., 2013a).

Despite the professionalisation of both domestic and international female cricket teams in recent years, only Stuelcken and colleagues (2007; 2008a; 2008b; 2009; 2010a; 2010b) have performed any investigations on female fast bowlers. These investigations have compared the anthropometric characteristics of males and females, the kinematic parameters associated with injury versus performance, or the kinetics of female fast bowling. To date there still remains a 'total lack of published biomechanical research' on the performance of female cricketers (Bartlett et al., 1996, Munro and Christie, 2018).

There is substantial evidence that males throw with a greater velocity than females in a number of throwing activities (Atwater, 1979; Thomas \& French, 1985; Thomas \& Marzke, 1992; Mero et al., 1994; Thomas et al., 1994; van den Tillaar \& Ettema, 2004; Chu et al., 2009; Thomas et al., 2010; van den Tillaar \& Cabri, 2012). The difference in throwing performance between males and females is considered multifactorial with strength and anthropometric components affecting technique and performance (Atwater, 1979; Thomas \& French, 1985; Thomas \& Marzke, 1992; van den Tillaar \& Ettema, 2004). For standing throws, such as those employed in baseball and handball, the difference in performance has been shown to be due to differences in strength and anthropometric components between males and females rather than differences in technique (Van den Tillaar \& Ettema. 2004; van den Tillaar \& Cabri, 2012; Chu et al., 2009). In javelin, a throw which incorporates a run-up similar to cricket fast bowling (Bartlett et al., 1996), elite male and female javelin throwers have been shown to employ different segment sequencing and joint angular motions (Liu et al., 2010). The main difference observed was that the male throwers were quicker with their rear leg extension motion than elite female javelin throwers - a technique which coaching literature suggests is associated with maintaining the kinetic energy accumulated during the run-up (Gorsksi, 1980). A similar motion was found by Ferdinands et al. (2014) when analysing eighteen young male fast bowlers, where the bowlers with quicker rear leg extension kinematics tended to produce faster bowling wrist speeds.

Due to the lack of previous research on female fast bowling, current fast bowling coaching literature has been informed by studies investigating optimal technique in male fast bowlers. This approach assumes that optimal fast bowling technique is the same for males and females as has been shown to be the case in standing throws (Chu et al., 2009; van den Tillaar \& Cabri, 2012). Given that differences have been observed in the segmental sequencing and joint angular motions between male and female javelin throwers, a throw with a similar run-up to cricket albeit with different objectives, this assumption may not be appropriate. The aim of this study is to compare the performance (ball release speed) and the kinematics of the fast bowling action between elite male and female fast bowlers to determine whether differences in techniques exist.

\section{METHODS}

\section{Data Collection}

20 female (mean \pm SD: age: $19.9 \pm 3.2$ years; height: $1.67 \pm 0.07 \mathrm{~m}$; body mass: $64.4 \pm 8.8 \mathrm{~kg}$ ) and 20 male (mean \pm SD: age: $20.1 \pm 2.6$ years; height: $1.88 \pm 0.08 \mathrm{~m}$; body mass: $81.5 \pm 7.1 \mathrm{~kg}$ ) elite fast bowlers participated in the study. All bowlers were either a member of the senior, A or U19 cricket national team, or a current professional 
identified as having the potential to play international cricket within the next 5 years. All bowlers were deemed fit to bowl by their County or National team physiotherapist and conducted a thorough warm-up before data collection. The testing procedures were explained to the bowlers prior to participation in accordance with Loughborough University ethical guidelines, a pre-selection medical questionnaire was completed, and informed consent obtained.

Kinematic data were collected at the ECB National Cricket Performance Centre at Loughborough University, an indoor practice facility where bowlers are able to use a full length run-up on a standard sized artificial cricket pitch. Forty-seven $14 \mathrm{~mm}$ retroreflective markers were attached to each bowler positioned over bony landmarks in accordance with the marker set used by Worthington et al. (2013a). An additional 15 $x 15 \mathrm{~mm}$ reflective patch was placed on the ball, using 3M Scotch-Lite reflective tape, to enable ball release speed and the moment of release to be calculated. Six maximal velocity deliveries of a good length were recorded using an 18 camera (MX13) Vicon Motion Analysis System (OMG PLC, Oxford, UK) operating at $300 \mathrm{~Hz}$. A static trial was performed for each bowler allowing body segment lengths and a neutral spine position to be calculated (Ranson et al., 2008). Ninety-five anthropometric measurements were also taken, enabling subject-specific segmental inertia parameters to be determined for each bowler using the mathematical model of Yeadon (1990).

\section{Data processing}

Three bowling trials for each bowler (maximum ball velocity with minimal marker loss) were manually labelled and processed within Vicon's software (OMG PLC, Oxford, UK). The marker trajectories were filtered using a recursive fourth-order lowpass Butterworth filter with a cut-off frequency of $30 \mathrm{~Hz}$ (Winter, 1990). The key instants of back foot contact (BFC), front foot contact (FFC) and ball release (BR) were identified manually using the motions of the markers throughout the action. BFC was defined as the first frame in which the back foot's motion was visually observed to change due to contact with the ground during the delivery stride (Worthington et al., 2013a). FFC corresponded to the first frame in which the front foot contacted the ground (Worthington et al., 2013a). BR was identified using the time history of the distance between the ball and the wrist joint centre. The frame corresponding to ball release was defined as the first frame in which this distance increased by more than $20 \mathrm{~mm}$ relative to the distance in the previous image (Worthington et al., 2013a).

Three-dimensional joint centre time histories for the ankle, knee, shoulder, elbow and wrists were calculated from the pair of markers placed medio-lateral of each joint (anterior-posterior for the shoulder) such that their midpoint coincided with the joint centre (Worthington et al., 2013a). The hip joint centre time histories were calculated from markers placed on the left and right anterior and posterior superior iliac spine (Davis et al., 1991). Joint centres for the lower and upper trunk as well as the head and neck segments were calculated using the four pelvis markers, in addition to the markers on the distal and proximal ends of the sternum and spinous processes of L1, T10 and C7 (Ranson et al., 2008).

Local reference frames were defined comprising a three-dimensional full-body 18-segment representation of a bowler (head and neck; upper trunk; lower trunk; pelvis; $2 \times$ arm; $2 \times$ lower-arm; $2 \times$ hand; $2 \times$ upper-leg $2 \times$ lower-leg; and $2 \times$ twosegment foot). Reference frames were defined using three markers on each segment, allowing segment orientations and joint angles to be calculated. The z-axis pointed upwards along the longitudinal axis of the segment, the $x$-axis pointed towards the 
bowler's right with the y-axis pointing forwards. Similarly, a global coordinate system was defined with the $y$-axis pointing down the wicket, towards the batsman, the $x$-axis pointing to the right and the z-axis pointing vertically upwards. Joint angles were calculated as Cardan angles, defined as a parent-child coordinate system. This defines the rotation applied to the parent coordinate system (proximal segment) to bring it into coincidence with the coordinate system of the child segment (distal segment). Rotation angles were calculated using an xyz sequence, corresponding to flexion-extension, abduction-adduction, and longitudinal rotation, respectively (Worthington et al., 2013a).

Ball release speed was calculated over a period of 10 frames $(0.033 \mathrm{~s})$ from the instant of BR using the equations of constant acceleration. The delivery stride length was calculated as the distance between the front and back foot ankle joint centres at FFC and was expressed as a percentage of the bowler's standing height. The front and rear plant angles were calculated by projecting the centre of mass (COM) location and the respective ankle joint centre onto a vertical plane and determining the angle between the vertical and the line joining the projected points (Worthington et al., 2013b). Similarly, the orientation of the trunk was calculated by projecting the midpoints of the hip and shoulder joint centres onto a vertical plane and determining the angle between the vertical and the line joining the projected points. The orientation of the shoulders and pelvis were calculated by projecting their respective joint centres onto the transverse plane (Worthington et al., 2013a). A bowler facing directly down the wicket (front-on) was defined to have a shoulder and pelvis orientation angle of $270^{\circ}$, a side-on position corresponded to an orientation angle of $180^{\circ}$ (Portus et al., 2004). The pelvis-shoulder separation angle was calculated as the difference between pelvis and shoulder orientation angles.

The knee, hip, and shoulder angles corresponded to flexion/extension of each joint $\left(\right.$ anatomical position $=180^{\circ}$, flexion $<180^{\circ}$ ). Similarly, the upper trunk angle corresponded to flexion/extension between the upper and lower trunk segments (anatomical position $=180^{\circ}$, flexion $<180^{\circ}$ ) and was normalised using the neutral position of the spine calculated from a static trial (Ranson et al., 2008).

\section{Data Analysis}

Fifty-five parameters were determined for each bowling trial, defining key performance and kinematic aspects of the fast bowling technique (Table 1 and Table 2).

All statistical analyses were performed within SPSS v.23 (SPSS Corporation, USA). The variation observed in each technique parameter (including ball release speed) was assessed using analysis of variance (ANOVA). The between-trial variability was compared with the between-bowler variability and was found to be much smaller. In particular, the between-trial standard deviations of the observations ranged from $1.1 \%$ to $13.6 \%$ (mean: $4.9 \%$ ) of the between-bowler variation for the parameters calculated in this study. This corresponded to an intra-class correlation coefficient (ICC) of 0.94-0.99 (mean: 0.98) which indicates good between-trial repeatability for the kinematic parameters calculated. Consequently, the three trials analysed were averaged for each parameter to provide representative data for each bowler (Worthington et al., 2013a).

To compare the performance (ball release speed) and kinematics between the male and female fast bowlers a student t-test for independent variables was used. An alpha value of 0.05 was used to define significance (Sinclair et al., 2013). If the assumption of normality was violated for any of the parameters, the non-parametric 
Mann-Whitney $U$ test was performed instead. Similarly, if the assumption that the variances are homogeneous was violated for any of the parameters, the Welch t-test was used. If the differences were significant, Cohen's d was calculated to determine the effect size of the difference.

\section{RESULTS}

The female fast bowlers produced significantly slower ball release speeds compared to their male counterparts $(27.9 \pm 1.4 \mathrm{~m} / \mathrm{s}$ vs. $34.9 \pm 1.7 \mathrm{~m} / \mathrm{s}, \mathrm{P}<0.01$; ES $>0.80)$. On average the female fast bowlers were also significantly shorter (1.67 \pm $0.07 \mathrm{~m}$ vs $1.88 \pm 0.08 \mathrm{~m}, \mathrm{P}<0.01 ; \mathrm{ES}>0.80)$ and lighter $(64.4 \pm 8.8 \mathrm{~kg}$ vs. $81.5 \pm 7.1$ $\mathrm{kg}, \mathrm{P}<0.01$, ES $>0.80$ ) than the male fast bowlers, even though they were of a comparable age $(19.9 \pm 3.2$ years vs. $20.1 \pm 2.6$ years, $P>0.05)$.

Ten significant differences with a large effect size $(>0.8)$ and one significant difference with a moderate effect size (>0.5), were observed in the kinematic parameters calculated at key instances of the fast bowling action (Table 1). At the instance of BFC, the female bowlers had lower horizontal COM velocities, less flexed front hip angles, and less extended upper trunk angles (Table 1). A further two parameters were significantly different at FFC with females having lower downward COM velocities and larger front leg plant angles (Table 1). The largest number of differences in the discrete parameters was at BR where differences in six of the eleven parameters were found. Females had on average lower normalised ball release heights, slower horizontal COM velocities, more front-on pelvis and shoulder orientations, more flexed upper trunk angles and more delayed bowling arm circumduction (Table 1).

Table 1 - Group means and standard deviations for selected position and velocity parameters at the key instant of the fast bowling action for male and female bowlers

\begin{tabular}{|c|c|c|c|c|c|c|}
\hline \multirow[b]{2}{*}{ parameters } & \multicolumn{2}{|c|}{ BFC } & \multicolumn{2}{|c|}{ FFC } & \multicolumn{2}{|c|}{ BR } \\
\hline & males & females & Males & females & males & females \\
\hline ball release speed $(\mathrm{m} / \mathrm{s})$ & & & & & $34.9 \pm 1.7^{* a}$ & $27.9 \pm 1.4$ \\
\hline ball release height (\% of standing height) & & & & & $112.8 \pm 4.1^{* a}$ & $109.7 \pm 3.0$ \\
\hline rear plant angle $\left({ }^{\circ}\right)$ & $-7.13 \pm 5.9$ & $-8.14 \pm 5.5$ & $39.1 \pm 2.8$ & $39.3 \pm 4.2$ & & \\
\hline front plant angle $\left(^{\circ}\right)$ & & & $-33.8 \pm 3.2 * a$ & $-37.4 \pm 2.8$ & & \\
\hline delivery stride length (\% of standing height) & & & $78.5 \pm 7.2$ & $76.3 \pm 7.8$ & & \\
\hline horizontal COM velocity (m/s) & $5.76 \pm 0.58 * a$ & $5.31 \pm 0.46$ & $5.23 \pm 0.54$ & $4.93 \pm 0.48$ & $3.69 \pm 0.40 * b$ & $3.36 \pm 0.52$ \\
\hline vertical COM velocity $(\mathrm{m} / \mathrm{s})$ & $-1.58 \pm 0.33$ & $-1.44 \pm 0.23$ & $-1.25 \pm 0.36^{* a}$ & $-0.94 \pm 0.23$ & $0.57 \pm 0.27$ & $0.52 \pm 0.21$ \\
\hline trunk orientation $\left({ }^{\circ}\right)$ & $-6.40 \pm 7.36$ & $-3.14 \pm 9.40$ & $-4.40 \pm 4.59$ & $-2.05 \pm 5.79$ & $41.7 \pm 4.7$ & $43.0 \pm 8.6$ \\
\hline pelvis orientation $\left({ }^{\circ}\right)$ & $219.3 \pm 14.9$ & $219.6 \pm 14.3$ & $231.9 \pm 8.5$ & $237.1 \pm 12.8$ & $288.0 \pm 10.6^{* a}$ & $298.2 \pm 9.0$ \\
\hline shoulder orientation $\left({ }^{\circ}\right)$ & $231.5 \pm 13.7$ & $233.5 \pm 19.6$ & $200.9 \pm 6.2$ & $205.0 \pm 12.0$ & $310.4 \pm 15.0^{* a}$ & $322.8 \pm 13.3$ \\
\hline pelvis-shoulder separation angle $\left({ }^{\circ}\right)$ & $12.2 \pm 12.5$ & $13.9 \pm 19.0$ & $-31.1 \pm 8.5$ & $-32.1 \pm 8.8$ & $22.4 \pm 10.0$ & $24.6 \pm 11.3$ \\
\hline back knee angle $\left(^{\circ}\right)$ & $149.4 \pm 11.1$ & $148.3 \pm 11.6$ & $138.7 \pm 9.6$ & $137.3 \pm 13.9$ & & \\
\hline front knee angle $\left(^{\circ}\right)$ & $114.8 \pm 32.4$ & $113.5 \pm 28.7$ & $164.1 \pm 6.1$ & $166.7 \pm 7.0$ & $167.3 \pm 18.8$ & $172.5 \pm 24.9$ \\
\hline back hip angle $\left(^{\circ}\right)$ & $148.2 \pm 11.9$ & $146.8 \pm 13.5$ & $186.0 \pm 8.0$ & $187.1 \pm 13.7$ & $146.6 \pm 11.0$ & $139.9 \pm 18.3$ \\
\hline front hip angle $\left(^{\circ}\right)$ & $105.6 \pm 19.5^{* a}$ & $123.0 \pm 21.5$ & $133.0 \pm 9.3$ & $130.9 \pm 14.3$ & $117.3 \pm 10.5$ & $116.6 \pm 12.0$ \\
\hline upper trunk angle $\left({ }^{\circ}\right)$ & $185.2 \pm 8.4^{* a}$ & $177.2 \pm 7.1$ & $185.3 \pm 7.6$ & $181.4 \pm 7.4$ & $159.5 \pm 7.8^{* a}$ & $153.1 \pm 7.7$ \\
\hline bowling shoulder angle $\left(^{\circ}\right)$ & & & $331.2 \pm 22.1$ & $337.5 \pm 17.2$ & $219.4 \pm 15.3^{* a}$ & $233.9 \pm 18.1$ \\
\hline
\end{tabular}

* Significant difference between males and females using an alpha value of 0.05 .

a large effect size $\geq 0.80,{ }^{b}$ medium effect size $\geq 0.50$ (Cohen's $d$ ) 
Three significant differences were found in the parameters calculated in the transitions between BFC and FFC, FFC and BR, and BFC and BR. Although no significant difference was found for the time between BFC and BR, significant differences were found for the time between BFC and FFC and between FFC and BR with moderate $(>0.5)$ and large $(>0.8)$ effect sizes respectively (Table 2$)$. Male bowlers were found to spend significantly more time between BFC and FFC, but significantly less time between FFC and BR compared to female bowlers. The only other significant difference with a large effect size $(>0.8)$ between males and females was the rotation of the pelvis orientation between BFC and BR (Table 2). More rotation of the pelvis orientation occurred in female bowlers between BFC and BR compared to males.

Table 1 - Group means and standard deviations for selected time and displacement parameters between the key instant of the fast bowling action for male and female bowlers.

\begin{tabular}{|c|c|c|c|c|c|c|}
\hline \multirow[b]{2}{*}{ parameters } & \multicolumn{2}{|c|}{ BFC to FFC } & \multicolumn{2}{|c|}{ FFC to BR } & \multicolumn{2}{|c|}{ BFC to BR } \\
\hline & males & females & males & females & males & females \\
\hline time (ms) & $191.9 \pm 31.3^{* b}$ & $172.3 \pm 27.6$ & $103.3 \pm 11.2 * a$ & $127.7 \pm 11.0$ & $295.2 \pm 33.4$ & $300.1 \pm 29.8$ \\
\hline minimum back knee angle $\left(^{\circ}\right)$ & $117.3 \pm 11.3$ & $113.8 \pm 10.1$ & & & & \\
\hline back knee flexion $\left({ }^{\circ}\right)$ & $32.1 \pm 11.2$ & $34.5 \pm 10.6$ & & & & \\
\hline back knee extension $\left({ }^{\circ}\right)$ & $21.5 \pm 8.0$ & $23.5 \pm 11.0$ & & & & \\
\hline minimum front knee angle $\left(^{\circ}\right)$ & & & $154.9 \pm 13.3$ & $154.0 \pm 15.8$ & & \\
\hline front knee flexion $\left({ }^{\circ}\right)$ & & & $9.2 \pm 10.8$ & $12.7 \pm 13.9$ & & \\
\hline front knee extension $\left({ }^{\circ}\right)$ & & & $12.4 \pm 8.6$ & $18.4 \pm 13.3$ & & \\
\hline trunk flexion $\left({ }^{\circ}\right)$ & & & $31.0 \pm 8.3$ & $35.5 \pm 7.3$ & & \\
\hline pelvis orientation rotation $\left(^{\circ}\right)$ & & & & & $68.7 \pm 15.3^{* b}$ & $78.6 \pm 15.2$ \\
\hline shoulder orientation rotation $\left({ }^{\circ}\right)$ & & & & & $78.9 \pm 19.2$ & $89.3 \pm 21.1$ \\
\hline minimum pelvis orientation $\left({ }^{\circ}\right)$ & & & & & $203.6 \pm 13.3$ & $209.5 \pm 14.4$ \\
\hline minimum shoulder orientation $\left({ }^{\circ}\right)$ & & & & & $194.9 \pm 6.8$ & $201.1 \pm 12.4$ \\
\hline minimum pelvis-shoulder separation $\left({ }^{\circ}\right)$ & & & & & $39.6 \pm 9.6$ & $45.1 \pm 13.4$ \\
\hline
\end{tabular}

* Significant difference between males and females using an alpha value of 0.05 .

${ }^{a}$ large effect size $\geq 0.80,{ }^{b}$ medium effect size $\geq 0.50$ (Cohen's $d$ )

\section{DISCUSSION}

The current study investigated the assumption that the optimal technique for ball release speed in fast bowling is the same for males and females, similar to standing throws (Chu et al., 2009; van den Tillaar \& Cabri, 2012), by comparing fast bowling performance and technique characteristics between male and female fast bowlers. The results of this research found significant differences in ball release speed and key technique parameters which may indicate that the female fast bowlers in this study potentially use a different technique to generate ball release speed.

Fast bowling performance is considered to be reliant on the linear momentum generated during the run-up due to the restrictions imposed by delivering the ball with a straight arm (Chapman, 2008). Previous research has found that faster ball release speeds are linked with faster run-up speeds (Davis and Blanksby, 1976a; Elliott et al., 1986; Worthington et al., 2013a). This study found that on average the female fast bowlers had significantly slower horizontal COM velocities than their male counterparts (Table 1). This difference remained throughout the fast bowling action with a significant difference, similar in magnitude, also found at BR (Table 1). Theoretically however, even if female fast bowlers were able to match the run-up speeds of the male fast bowlers as in javelin (5.6 m/s for both; Mero et al., 1994) and produce the same 
kinematics for each segment, they would likely produce slower ball release speeds since females are likely to have proportionally shorter torso and arm lengths due to their significantly shorter body heights (Stuelcken et al., 2007).

At BFC, the male fast bowlers were observed to have a higher front leg (smaller front hip flexion angle) and more extended upper trunk angles (more leaned back) compared to the female bowlers (Table 1). Chu et al. (2009) discussed that female pitchers in baseball may have a lower front knee lift compared to male pitchers as they lack the muscular strength to extend the trunk and keep balance with a higher front knee. It is speculated cricket fast bowling is similar and female fast bowlers may also lack the muscular strength required to extend the trunk and keep balance with a higher front knee.

The significantly longer transition between BFC and FFC is most likely caused by the BFC position during this transition for male bowlers compared to female bowlers. The male bowlers started in a position at BFC which required them to travel through a larger range of motion at the trunk and hip compared to the female bowlers (Table 1). The larger vertical COM velocities and larger plant angles at FFC (Table 1) suggest that female fast bowlers may have a difference in the timing of the extension of the rear leg between BFC and FFC. Although the cause is not obvious and should be a topic of further study, an earlier timing of the extension of the rear leg has previously been identified as an important technique parameter for performance in cricket (Ferdinands et al., 2014). While in javelin, a difference in timing was found between genders where male throwers extend their rear leg earlier than female throwers (Liu et al., 2010). An earlier extension of the rear leg pushes the COM forward horizontally rather than it continuing its downward path, a technique which javelin coaching literature suggests is associated with maintaining the linear momentum during the transition from BFC to FFC (Gorsksi, 1980).

The significant difference in the time between FFC and BR suggests that there may be a difference in the amount of angular momentum generated during this transition. The more angular momentum generated the faster the trunk will rotate and the shorter this transition. This movement was faster in male bowlers compared to female bowlers, similar to other throwing activities such as baseball (Chu et al., 2009) and javelin (Liu et al., 2010). The times were shorter however for cricket compared with javelin and baseball (males: 103 ms vs. 115 ms vs. 141 ms; females: 128 ms vs. $137 \mathrm{~ms}$ vs. $163 \mathrm{~ms}$ ), although care is required when comparing across studies due to methodological differences in identifying the key instances. The cause of the difference in time between FFC and BR for males compared to females is still unknown and should be the focus of future research.

At $\mathrm{BR}$, the female fast bowlers had more front-on pelvis and shoulder orientations as well as more flexed upper trunk angles with more delayed bowling arm circumduction than male fast bowlers (Table 1). These differences, coupled with the significant difference in rotation of the pelvis orientation between BFC and BR (Table 2), suggest that females use a rotational sequencing motion more akin to throwing than male fast bowlers. Previous research has shown that the fastest male bowlers generate ball release speed by maximising their whole body linear momentum during the run-up and converting it to whole body angular momentum about the COM (Worthington et al., 2013a). When this is done most efficiently the time between FFC and BR is reduced. It is hypothesised that this time is shorter than the time required for the large rotator muscles in the trunk to accelerate the pelvis and torso segments around the longitudinal axis and increase ball release speed. When the time between FFC and BR is increased above this threshold, the differences in technique 
characteristics at BR between male and female bowlers in this study suggest that a more rotational sequencing motion occurs. It is speculated this happens as the large rotator muscles have more time to generate power and this can be utilised to contribute to ball release speed, similar to other throws (Chapman, 2008). The cause and effect of these two mechanisms on ball release speed is unknown and should be the topic of future research.

There are some limitations with the study. Firstly, although both groups of bowlers are considered elite, due to the recent professionalisation of the female game it is likely that the male fast bowlers have had considerably more experience and time within a professional training environment throughout their development than the females. In addition, whilst both groups represent the sample from which they have been recruited in each gender, the population size of professional male cricketers is much larger compared to professional females. This could potentially skew the participants in talent and result in a sample size bias (Chu et al., 2009). Finally, an adjustment to the alpha level due to multiple comparisons was not applied since it increases the incidence of Type 2 errors, which have previously been proposed to be a more substantial threat to exploratory analyses than Type 1 errors (Sinclair et al., 2013). Adopting this approach however requires the findings to be considered cautiously as an increased risk of Type 1 errors occurring remains.

In conclusion, the female fast bowlers in this study had slower run-up speeds than the male bowlers and subsequently less whole body linear momentum. Furthermore, during the transition from BFC to FFC, the male bowlers adopted a technique which more efficiently maintained the whole body linear momentum developed during the run-up. As a result the male bowlers had more whole body linear momentum to convert into whole body angular momentum about the mass centre between FFC and BR. The kinematic differences between FFC and BR indicate that, in contrast to the male fast bowlers, the amount of whole body angular momentum generated by the female fast bowlers was insufficient to solely generate ball speed. Instead the female bowlers adopted a technique more akin to throwing where ball speed is contributed to by both the whole body angular momentum and the large rotator muscles used to rotate the pelvis and torso segments about the longitudinal axis. The results of this investigation are likely to be useful in the coaching and conditioning of female fast bowlers and potentially junior bowlers with developing anthropometric and strength parameters. Future research should attempt to understand the limitations anthropometric and strength constraints may have on fast bowling performance.

\section{REFERENCES}

Atwater, A.E. (1979). Biomechanics of overarm throwing movements and of throwing injuries. Exercise and Sport Sciences Reviews, 7, 43-86.

Bartlett, R.M., Stockill, N.P., Elliott, B.C., \& Burnett, A.F. (1996). The biomechanics of fast bowling in men's cricket: A review. Journal of Sports Sciences, 14, 403-424.

Burden, A.M., \& Bartlett, R.M. (1990a). A kinematic comparison between elite fast bowlers and college fast-medium bowlers. Proceedings of the Sports Biomechanics Section of the British Association of Sports Sciences, 15, 41-46.

Burden, A.M., \& Bartlett, R.M. (1990b). A kinematic investigation of elite fast and fast medium cricket bowlers. In Proceedings of the VIIIth International Symposium of the Society of Biomechanics in Sports (edited by M. Nosek, D. Sojka, W. Morrisson and P. Susanka), pp.41-46. Prague: Consport. 
Chapman, A.E. (2008). Biomechanical analysis of fundamental human movements. Champaign, IL: Human Kinetics.

Chu, Y., Fleisig, G.S., Simpson, K.J., \& Andrews, J.R. (2009). Biomechanical comparison between elite female and male baseball pitchers. Journal of Applied Biomechanics, 25, 22-31.

Davis, K., \& Blanksby, B. (1976a). The segmental components of fast bowling in cricket. Australian Journal for Health, Physical Education and Recreation, 71(suppl.), 6-8.

Davis, K., \& Blanksby, B. (1976b). A cinematographical analysis of fast bowling in cricket. Australian Journal for Health, Physical Education and Recreation, 71 (suppl.), 9- 15.

Davis, R.B., Ounpuu, S., \& Tyburski, D. (1991). A gait analysis data collection and reduction technique. Human Movement Science, 10, 575-587.

Elliott, B.C., Foster, D., \& Gray, S. (1986). Biomechanics and physical factors affecting fast bowling. Australian Journal of Science and Medicine in Sport, 18, 16-21.

Ferdinands, R.E.D., Sinclair, P.J., Stuelcken, M.C., \& Greene, A. (2014). Rear leg kinematics and kinetics in cricket fast bowling. Sports Technology, 7, 52-61.

Foster, D., John, D., Elliott, B., Ackland, T., \& Fitch, K. (1989). Back injuries to fast bowlers in cricket: A prospective study. British Journal of Sports Medicine, 23, 150154.

Gorski, J.P. (1980). Developing the javelin soft-step. Track Technique, 81, 2581-2582.

Liu, H., Leigh, S., \& Yu, B. (2010). Sequences of upper and lower extremity motions in javelin throwing. Journal of Sports Sciences, 28, 1459-1467.

Munro C.E., \& Christie C.J. (2018). Research directions for the enhancement of women's cricket. International Journal of Sports Science and Coaching, 2018, 0(0),1-5. https://doi.org/10.1177/1747954118764102

Mero, A., Komi, P.V., Korjus, T., Navarro, E., \& Gregor, R.J. (1994). Body segment contributions to javelin throwing during final thrust phases. Journal of Applied Biomechanics, 10, 166-177.

Portus, M.R., Mason, B.R., Elliott, B.C., Pfitzner, M.C., \& Done, R.P. (2004). Technique factors related to ball release speed and trunk injuries in high performance cricket fast bowlers. Sports Biomechanics, 3, 263-283.

Ranson, C.A., Burnett, A.F., King, M.A., Patel, N., \& O'Sullivan, P.B. (2008). The relationship between bowling action classification and three-dimensional lower trunk motion in fast bowlers in cricket. Sports Biomechanics, 3, 263-283.

Sinclair, J.K., Taylor, P.J., \& Hobbs, S.J. (2013). Alpha level adjustments for multiple dependent variable analyses and their application - A review. International Journal of Sports Science and Engineering, 7, 17-20.

Stuelcken, M., Pyne, D., \& Sinclair, P. (2007). Anthropometric characteristics of elite cricket fast bowlers. Journal of Sports Sciences, 25, 1587-1597.

Stuelcken, M.C., Ginn, K.A., \& Sinclair, P.J. (2008a). Shoulder strength and range of motion in elite female cricket fast bowlers with and without a history of shoulder pain. Journal of Science and Medicine in Sport, 11, 575-580.

Stuelcken, M.C., Ginn, K.A., \& Sinclair, P.J. (2008b). Musculoskeletal profile of the lumbar spine and hip regions in cricket fast bowlers. Physical Therapy in Sport, 9, 82-88. 
Stuelcken, M.C., \& Sinclair, P.J. (2009). A pilot study of the front foot ground reaction forces in elite female fast bowlers. Journal of Science and Medicine in Sport, 12, 258-261.

Stuelcken, M.C., Ferdinands, R.E., \& Sinclair, P.J. (2010a). Three-dimensional trunk kinematics and low back pain in elite female fast bowlers. Journal of Applied Biomechanics, 26, 52-61.

Stuelcken, M.C., Sinclair, P.J., \& Ginn, K.A. (2010b). The shoulder distraction force in cricket fast bowling. Journal of Applied Biomechanics, 25, 373-377.

Thomas, J.R., \& French, K.E. (1985). Gender differences across age in motor performance: A meta-analysis. Psychological bulletin, 98, 260.

Thomas, J.R., \& Marzke, M.W. (1992). The development of gender differences in throwing: Is human evolution a factor. In R.W. Christina \& H.m. Eckert (Eds.), Enhancing human performance in sport: New concepts and developments (pp. 6076). Champaign, IL: Human Kinetics.

Thomas, J.R., Michael, D., \& Gallagher, J.D. (1994). Effects of training on gender differences in overhand throwing: A brief quantitative literature analysis. Research Quarterly for Exercise and Sport, 65, 67-71.

Thomas, J.R., Alderson, J.A., Thomas, K.T., Campbell, A.C., \& Elliott, B.C. (2010). Developmental gender differences for overhand throwing in aboriginal Australian children. Research Quarterly for Exercise and Sport, 81, 432-441.

Van den Tillaar, R., \& Ettema, G. (2004). Effect of body size and gender in overarm throwing performance. European Journal of Applied Physiology, 91, 413-418.

Van Den Tillaar, R., \& Cabri, J.M. (2012). Gender differences in the kinematics and ball velocity of overarm throwing in elite team handball players. Journal of Sports Sciences, 30, 807-813.

Winter, D.A. (1990). Biomechanics and motor control of human movement. New York: Willey.

Worthington, P.J., King, M.A., \& Ranson, C.A. (2013a). Relationships between fast bowling technique and ball release speed in cricket. Journal of Applied Biomechanics, 29, 78 - 84.

Worthington, P.J., King, M.A., \& Ranson, C.A. (2013b). The influence of cricket fast bowlers' front leg technique on peak ground reaction forces. Journal of Sports Sciences, 31, 434-441.

Yeadon, M.R. (1990). The simulation of aerial movement - II. A mathematical inertia model of the human body. Journal of Biomechanics, 23, 67-74. 